\title{
Online Firefighting on Trees
}

\author{
Pierre Coupechoux ${ }^{1}$, Marc Demange ${ }^{2}$, David Ellison*2, and \\ Bertrand Jouve ${ }^{\dagger 3}$ \\ ${ }^{1}$ LAAS-CNRS, Université de Toulouse, CNRS, Toulouse, France, \\ pierre.coupechoux@laas.fr \\ ${ }^{2}$ RMIT University, School of Science, Melbourne, Australia \\ marc.demange@rmit.edu.au,david.ellison2@rmit.edu.au \\ ${ }^{3}$ FRAMESPA and IMT, CNRS, Université de Toulouse, Toulouse, \\ France bertrand.jouve@cnrs.fr
}

\begin{abstract}
In the FIREFighter problem, introduced by Hartnell in 1995, a fire spreads through a graph while a player chooses which vertices to protect in order to contain it. In this paper, we focus on the case of trees and we consider as well the Fractional Firefighter game where the amount of protection allocated to a vertex lies between 0 and 1 . We introduce the online version of both Firefighter and Fractional Firefighter, in which the number of firefighters available at each turn is revealed over time. We show that the greedy algorithm on finite trees, which maximises at each turn the amount of vertices protected, is $1 / 2$-competitive for both online versions; this was previously known only in special cases of FIREFIGHTER. We also show that, for FiREFighteR, the optimal competitive ratio of online algorithms ranges between $1 / 2$ and the inverse of the golden ratio. The greedy algorithm is optimal if the number of firefighters is not bounded and we propose an optimal online algorithm which reaches the inverse of the golden ratio if at most 2 firefighters are available. Finally, we show that on infinite trees with linear growth, any firefighter sequence stronger than a non-zero periodic sequence is sufficient to contain the fire, even when revealed online.
\end{abstract}

\section{Introduction and Definitions}

Since it was formally introduced by B. Hartnell in 1995 ([1], cited in [2]) the firefighting problem - FIREFIGHTER - has raised the interest of many researchers. While this game started as a very simple model for fire spread and containment

*corresponding author.

${ }^{\dagger}$ We acknowledge the support of GEO-SAFE, H2020-MSCA-RISE-2015 project \# 691161. 
problems for wildfires, it can also represent any kind of threat able to spread sequentially in a network (diseases, viruses, rumours, flood ...).

It is a deterministic discrete-time one-player game defined on a graph. In the beginning, a fire breaks out on a vertex and at each step, if not blocked, the fire spreads to all adjacent vertices. In order to contain the fire, the player is given a number $f_{i}$ of firefighters at each turn $i$ and can use them to protect vertices which are neither burning nor already protected. The game terminates when the fire cannot spread any further. In the case of finite graphs the aim is to save as many vertices as possible, while in the infinite case, the player wins if the game finishes, which means that the fire is contained.

This problem and its variants give rise to a generous literature; the reader is referred to [2] for a broad presentation of the main research directions. A significant amount of theoretical work deals with its complexity and approximability behaviour in various classes of graphs $[3,4,5,6]$ and recently its parametrised complexity (e.g. [7]). It is known to be very hard, even in some restrictive cases. In particular, the case of trees was revealed to be very rich and a lot of research focuses on it. The problem is NP-hard on finite trees [3], even in more restricted cases [6]. Regarding approximability results on trees, it was first shown to be $\frac{1}{2}$-approximated by a greedy strategy [8], improved to $1-\frac{1}{e}[5]$ and very recently to a polynomial time approximation scheme [9], which closes essentially the question of approximating firefighter problem in trees and motivates considering some generalisations. On general graphs the problem is hard to approximate within $n^{1-\varepsilon}$ [10]. A related research direction investigates integer linear programming models for the problem, especially on trees $[9,11,12]$. This line of research makes very natural a relaxed version where the amount of firefighters available at each turn is any non-negative number and the amount allocated to vertices lies between 0 and 1 . A vertex with a protection less than 1 is partially protected and its unprotected part can burn partially and transmit only its fraction of fire to the adjacent vertices. Thus, the $f_{i}$ may take any nonnegative value. This defines a variant game called Fractional Firefighter which was introduced in [13].

Online optimisation [14] is a generalisation of approximation theory which represents situations where the information arrives over the time and one needs to make irrevocable decisions. We propose an online version of both firefighter problems and consider first results on trees. In our model, the graph is known and the sequence of available firefighters is revealed online. We then refer to the usual case where $\left(f_{i}\right)_{i \geq 1}$ is known in advance as offline. To our knowledge, this is the first attempt at analysing online firefighter problems. Even though our motivation is mainly theoretical, this paradigm is particularly natural in emergency management where one has to make quick decisions despite lack of information. Any progress in this direction, even on simplified models, contributes to understanding how lack of information impacts the quality of the solution.

Given a tree $T$ rooted in $r, V(T)$ and $E(T)$ will denote the vertex set and the edge set of $T$, respectively. Given two vertices $v$ and $v^{\prime}, v \prec v^{\prime}$ denotes that $v$ is an ancestor of $v^{\prime}$ (or $v^{\prime}$ is a descendant of $v$ ) and $v \preceq v^{\prime}$ denotes that either 
$v=v^{\prime}$ or $v \prec v^{\prime}$.

For any vertex $v$, let $T[v]$ denote the sub-tree induced by $v$ and its descendants. Let $T_{i}$ denote the $i$-th level of $T$ rooted in $r$, where $\{r\}=T_{0}$. The height $h(T)$ of $T$, rooted in $r$, is the maximum length of a path from $r$ to a leaf. If $i>h(T)$, we have $T_{i}=\emptyset$.

If $T$ is finite, the weight $w(v)$ of a vertex $v$ is the number of vertices in its sub-tree (including $v$ ), i.e. $w(v)=|V(T[v])|$. When no ambiguity may occur, we will simply write $w_{v}=w(v)$. For any vertex $v \in T_{i}$ and any $i \leq j \leq i+h(T[v])$, we denote by $v_{j}$ a vertex of maximum weight $w_{v_{j}}$ in $T_{j} \cap V(T[v])$. While $v_{j}$ is not defined if $i+h(T[v])<j \leq h(T)$, we define $\bar{w}_{v_{j}}$ for all $j \leq h(T)$ and $v \in V(T)$ via: $\bar{w}_{v_{j}}=w_{v_{j}}$ if $j \leq i+h(T[v])$ and 0 otherwise. We denote by $B(T)$ the tree obtained from $T$ by fusing all vertices from levels 0 and 1 into a new root vertex $r_{B}$ : every edge $u_{1} u_{2} \in E(T)$ with $u_{1} \in T_{1}$ and $u_{2} \in T_{2}$ gives rise to the edge $r_{B} u_{2} \in E(B(T))$. For $k \leq h(T), B^{k}(T)$ will denote the $k^{\text {th }}$ iteration of $B$ applied to $T$ : all vertices from levels 0 to $k$ are fused into a single vertex denoted by $r_{B^{k}}$ which becomes the new root.

\section{The problems and preliminary results.}

\subsection{Firefighter and Fractional Firefighter on trees}

In this paper we only play the game on finite or infinite trees. An instance of the Fractional Firefighter on trees is defined by a triple $\left(T, r,\left(f_{i}\right)\right)$, where $T=(V(T), E(T))$ is a tree, $r \in V(T)$ is the root where the fire breaks out and $\left(f_{i}\right)_{i \geq 1}$ is the non-negative real firefighter sequence. Turn $i=0$ is the initial state where $r$ is burning and all other vertices are unprotected, and $i \geq 1$ corresponds to the different rounds of the game. At each turn $i \geq 1$ and for every vertex $v$, the player decides which amount $p(v)$ of protection to add to $v$. Throughout the game, for every vertex $v$ the part of $v$ which is burning is denoted by $b(v)$. Let us note that if $T$ is finite, the game will end in at most $h(T)$ turns where $h(T)$ is the length of a longuest path form the root to the leaves.

Solutions on trees have a very specific structure: at each turn $i$, the amounts of fire are non-increasing along any root to leaf path, which means that the fire will spread only towards the leaves. Note that for any solution which allocates a positive amount of protection at turn $i$ to a vertex $v \in T_{k}, k>i$, allocating the same amount of protection to $v$ 's father instead strictly improves the performance. So we may consider only algorithms that play in $T_{i}$ at turn $i$. For an optimal offline algorithm, this property was emphasised in [8]. So, for any vertex $v \in T_{i}$, the amounts of fire $b(v)$ and protection $p(v)$ on $v$ will not change after turn $i$.

A solution $p$ is characterised by the values $p(v), v \in V(T)$. For any solution $p$, while $p(v)$ represents the amount of protection received directly, vertex $v$ also receives protection through its ancestors, the amount of which is denoted by $P_{p}(v)=\sum_{v^{\prime} \prec v} p\left(v^{\prime}\right)$ (used in section 3). For any vertex $v$, we have the equality $p(v)+P_{p}(v)+b(v)=1$. 
Any solution $p$ for Firefighter or Fractional Firefighter will satisfy the constraints:

$$
\begin{cases}\sum_{v \in T_{i}} p(v) \leq f_{i} & (i) \\ \forall v, p(v)+P_{p}(v) \leq 1 & (i i)\end{cases}
$$

In [12], a specific boolean linear model has been proposed for solving FIREFighter on a tree $T$ involving these constraints. Solving Fractional FireFIGHTER on $T$ corresponds to solving the relaxed version of this linear programme.

\subsection{Online version}

We introduce online versions of Firefighter and Fractional Firefighter. The graph - a tree $T$ in this work - and the ignition vertex - the root $r$ - are known in advance but the firefighter sequence $\left(f_{i}\right)_{i>1}$ is revealed over time by a second player called adversary. At each turn $i$, the adversary reveals $f_{i}$ and then the player chooses where to allocate this ressource.

Let us consider an online algorithm $O A$ for one of the two problems and let us play the game on a finite tree $T$ until the fire stops spreading. The value $\lambda_{O A}$ achieved by the algorithm, defined as the amount of saved vertices, is measured against the best value performed by an algorithm knowing in advance the sequence $\left(f_{i}\right)$. In the present case, it is simply the optimal value of the offline instance, referred to as the offline optimal value, denoted by $\beta_{I}$ when considering the online Firefighter ( $I$ stands for "Integral") and $\beta_{F}$ for the online Fractional Firefighter. We will call $B o b$ such an algorithm able to see the future and guaranteeing the value $\beta_{I}$ or $\beta_{F}$ for online FirefighteR and Fractional Firefighter.

$O A$ is said to be $\gamma$-competitive, $\gamma \in] 0,1]$ for the online Firefighter (resp. Fractional Firefighter) if for every instance, $\frac{\lambda_{O A}}{\beta_{I}} \geq \gamma\left(\right.$ resp. $\left.\frac{\lambda_{O A}}{\beta_{F}} \geq \gamma\right) \cdot \gamma$ is also called the competitive ratio guaranteed by $O A$. An online algorithm will be called optimal if it guarantees the best possible competitive ratio.

Let us first note that one can reduce the problem to the case where $f_{1}>0$ :

Proposition 1. We can reduce online (Fractional) Firefighter on trees to instances where $f_{1}>0$.

Proof. If $f_{i}=0$ for all $i$ such that $1 \leq i \leq k$, then the instance $\left(T, r,\left(f_{i}\right)\right)$ is equivalent to the instance $\left(B^{k}(T), r_{B^{k}},\left(f_{i+k}\right)\right)$.

In the infinite case we do not define competitivness but only ask whether the fire can be contained by an online algorithm. Sections 3 and 4 deal with the finite case while section 5 deals with a class of infinite trees.

\section{Competitive analysis of a Greedy algorithm}

Greedy algorithms are usually very good candidates for online algorithms, sometimes the only known approach. Mainly two different greedy algorithms have 
been considered in the literature for FIREFIGHTER on a tree [2] and they are both possible online strategies in our set-up. The degree greedy strategy prioritises saving vertices of large degree; it has been shown in [6] that it cannot guarantee any approximation ratio on trees, even for a constant firefighter sequence. A second greedy algorithm was introduced in [8] for an integral sequence $\left(f_{i}\right)$, maximising at each turn the weight of the vertices protected. We generalise it to any firefighter sequence for both the integral and the fractional problems: at each turn $i$, the algorithm $G r$ solves the linear programme $\mathcal{P}_{i}$ with variables $x(v), v \in T_{i}$ and constraints:

$$
\mathcal{P}_{i}:\left\{\begin{array}{l}
\max \sum_{v \in T_{i}} x(v) w(v) \\
\sum_{v \in T_{i}} x(v) \leq f_{i} \\
\forall v, x(v)+P_{x}(v) \leq 1
\end{array}\right.
$$

An optimal solution of $\mathcal{P}_{i}$ is obtained by ordering vertices $\left\{v_{1}, \ldots, v_{\left|T_{i}\right|}\right\}$ of level $i$ by non-increasing weight and taking them one by one in this order and greedily assigning to vertex $v_{j}$ the value $x\left(v_{j}\right)=\min \left(f_{i}-\sum_{k<j} x\left(v_{k}\right), 1-\right.$ $\left.P_{x}\left(v_{j}\right)\right)$. Note that $G r$ is valid for both Firefighter and Fractional FireFIGHTER.

It was shown in [8] that the greedy algorithm on trees gives a $\frac{1}{2}$-approximation of the restriction of FIREFIGHTER when a single firefighter is available at each turn. They claim that this approximation ratio remains valid for a fixed number $D \in \mathbb{N}$ of firefighters at each turn. We extend this result to any firefighter sequence $\left(f_{i}\right)_{i \geq 1}$, integral or not. Since $G r$ is an online algorithm, the performance can also be seen as a competitive ratio for the online version.

Theorem 1. The greedy algorithm $G r$ is $\frac{1}{2}$-competitive for both online FIREFIGHTER and FRACTIONAL FIREFIGHTER on finite trees.

Proof. Let us first consider the fractional case with an online instance $\left(T, r,\left(f_{i}\right)\right)$ of Fractional Firefighter on a tree.

Let $x(v)$ and $y(v)$ be the amounts of firefighters placed on vertex $v$ by $G r$ and $B o b$, respectively. We have $\lambda_{G r}=\sum_{v} x(v) w(v)$ and $\beta_{F}=\sum_{v} y(v) w(v)$.

Recall that $P_{x}(v)=\sum_{v^{\prime} \prec v} x\left(v^{\prime}\right)$ and $P_{y}(v)=\sum_{v^{\prime} \prec v} y\left(v^{\prime}\right)$. We split $y(v)$ into two non-negative quantities, $y(v)=g(v)+h(v)$, with:

$g(v)=\min \left\{y(v), \max \left\{0, P_{x}(v)-P_{y}(v)\right\}\right\}$

and $h(v)=\max \left\{0, y(v)+\min \left\{0, P_{y}(v)-P_{x}(v)\right\}\right\}$.

We now claim that $\forall v^{\prime} \in T, \sum_{v \preceq v^{\prime}} g(v) \leq P_{x}\left(v^{\prime}\right)$ and prove it by induction.

Since $g(r)=0$, it holds for the root $r$. Assuming that the inequality holds for a vertex $v^{\prime}$, let $v^{\prime \prime}$ be a child of $v^{\prime}$. If $P_{x}\left(v^{\prime \prime}\right)-P_{y}\left(v^{\prime \prime}\right) \geq 0$, then we directly have:

$\sum_{v \prec v^{\prime \prime}} g(v)=\sum_{v \prec v^{\prime \prime}} g(v)+g\left(v^{\prime \prime}\right) \leq \sum_{v \prec v^{\prime \prime}} y(v)+\left(P_{x}\left(v^{\prime \prime}\right)-P_{y}\left(v^{\prime \prime}\right)\right)=P_{x}\left(v^{\prime \prime}\right)$.

Else $g\left(v^{\prime \prime}\right)=0$ and using $\sum_{v \preceq v^{\prime}} g(v) \leq P_{x}\left(v^{\prime}\right)$ and $P_{x}\left(v^{\prime \prime}\right) \geq P_{x}\left(v^{\prime}\right)$, the inequality holds for $v^{\prime \prime}$; which completes the proof of the claim.

Thus: $\sum_{v^{\prime}} \sum_{v \prec v^{\prime}} g(v) \leq \sum_{v^{\prime}} P_{x}\left(v^{\prime}\right)=\sum_{v^{\prime}} \sum_{v \prec v^{\prime}} x(v) \leq \sum_{v^{\prime}} \sum_{v \preceq v^{\prime}} x(v)$.

Since $w(v)=\sum_{v \preceq v^{\prime}} 1$, by inverting the sums on both sides, we obtain: 


$$
\sum_{v} g(v) w(v) \leq \sum_{v} x(v) w(v)=\lambda_{G r}
$$

Let us now consider the coefficients $h(v)$. We claim that the coefficients $h(v)$ with $v \in T_{i}$ satisfy the constraints $(i)$ and $(i i)$ of $\mathcal{P}_{i}$ : indeed for $(i)$, we have $h(v) \leq y(v)$ and $y$ satisfies constraint $(i)$. For $(i i)$ note that $h(v)+P_{x}(v)=$ $\max \left\{P_{x}(v), y(v)+\min \left\{P_{x}(v), P_{y}(v)\right\}\right\} \leq \max \left\{P_{x}(v), y(v)+P_{y}(v)\right\} \leq 1$.

Hence, $\forall i, \sum_{v \in T_{i}} h(v) w(v) \leq \sum_{v \in T_{i}} x(v) w(v)$ and therefore:

$$
\sum_{v \in T} h(v) w(v) \leq \sum_{v \in T} x(v) w(v)=\lambda_{G r}
$$

Finally, since $g(v)+h(v)=y(v)$, we conclude from eqs. (1) and (2) that $\beta_{F} \leq 2 \lambda_{G r}$. Hence the Greedy algorithm is $\frac{1}{2}$-competitive for the online FRACTIONAL Firefighter problem. Since the greedy algorithm gives an integral solution if $\left(f_{i}\right)$ has integral values and since $\beta_{F} \geq \beta_{I}$, it is also $\frac{1}{2}$-competitive for the Firefighter problem. This concludes the proof of theorem 1.

Conjecture 2.3 in [11] (which is also Conjecture 3.5 in [2]) claims that there is a constant $\rho$ such that the optimal value of FraCtionaL Firefighter is at most $\rho$ times the optimal value of Firefighter. It was supported by extensive experimental tests [11], but finding such a constant and proving the ratio is one of the open problems proposed in [2] (Problem 7). It was shown in [15] that such a constant must be greater than $\frac{e}{e-1}$. Theorem 1 can be expressed by $\lambda_{G r} \leq \beta_{I} \leq \beta_{F} \leq 2 \lambda_{G r}$, which shows that $\rho=2$ is such a constant:

Corollary 1. In Fractional Firefighter, the amount of vertices saved is at most twice the maximum number of vertices saved in FIREFIGHTER.

\section{Improved Competitive Algorithm for FiREFIGHTER}

In this section, we investigate possible improvements for online strategies for FiREFIGHTER on finite trees. Let $\varphi=\frac{1+\sqrt{5}}{2}$ denote the golden ratio, satisfying $\varphi^{2}=\varphi+1$ and $\frac{1}{\varphi}=\varphi-1$. For any integer $k \geq 2$, we denote

$$
\alpha_{I, k}=\inf _{T \in \mathcal{T}} \max _{O A \in \mathcal{A}_{L}} \min _{\left(f_{i}\right) \in \mathbb{N}^{N}, \sum_{i} f_{i} \leq k} \frac{\lambda_{O A}}{\beta_{I}},
$$

where $\mathcal{T}$ denotes the set of finite rooted trees and $\mathcal{A}_{L}$ the set of online algorithms for FirEFIGHTER on finite trees, be the best possible competitive ratio for online Firefighter on finite trees if at most $k$ firefighters are available. Note that the sequence $\left(\alpha_{I, k}\right)_{k}$ is non-increasing. Then, $\alpha_{I}=\inf _{k \geq 2} \alpha_{I, k}$ is the best possible competitive ratio for online Firefighter on finite trees. The index $I$ stands for Integral and refers to the problem FiREFighter. 
In what follows we give an optimal online algorithm for FIREFIGHTER on a finite tree in the case where at most two firefighters are available. Based on proposition 1 we may assume $f_{1}=1$ and such an online instance is characterised by when the second firefighter is presented.

Lemma 1. Let $a$ and $b$ be two vertices of maximum weights in $T_{1}$. If $\sum_{i} f_{i} \leq 2$, there is an optimal offline algorithm for FIREFIGHTER which places the first firefighter on either $a$ or $b$.

Proof. If the first firefighter is placed on $v \in T_{1} \backslash\{a, b\}$ by an optimal offline algorithm and since at most two firefighters are available, $\exists u \in\{a, b\}, T[u]$ burns completely. Then replacing $v$ by $u$ when assigning the first firefighter would produce another optimal solution (necessarily $w_{v}=w_{u}$ ).

We suppose $B o b$ has this property but note that even if $w_{a}>w_{b}$, he will not necessarily choose $a$; as illustrated by the graph $W_{1,10,20}$ (fig. 1 ): if the firefighter sequence is $(1,0,1,0, \ldots)$, then $B o b$ 's first move needs to be on $x$. Note also that, when the root is of degree at least 3 , the second firefighter is not necessarily in $V(T[a]) \cup V(T[b])$.

We now consider algorithm 1 and assume that the adversary will reveal at most two firefighters. The case where $f_{1}=2$ is trivial since an online algorithm can make the same decision as $B o b$ by assigning both firefighters to two unburnt vertices of maximum weights. So, we consider a binary firefighter sequence.

The algorithm works on an updated version $\widetilde{T}$ of the tree: if one vertex is protected, then the corresponding sub-tree is removed and all the burnt vertices are fused into the new root $\tilde{r}$ so that the algorithm always considers vertices of level 1 in $\widetilde{T}$. Before starting the online process, the algorithm computes all weights of vertices. Weights of unburnt vertices will not change when updating $\widetilde{T}$. The value of $h(\widetilde{T})$, required in line 6 can be computed during the initial calculation of weights and easily updated with $\widetilde{T}$. For clarity, we do not detail all update in the algorithm. The notation $\bar{w}_{v_{j}}$ used at line 6 is defined in section 1 .

Theorem 2. Algorithm 1 is a $\frac{1}{\varphi}$-competitive online algorithm for online FIREFIGHTER with at most two available firefighters. It is optimal for this case.

Proof. If the adversary does not present any firefighter before the turn $h(T)$, both algorithm 1 and $B o b$ cannot save any vertex and by convention one considers then that the competitive ratio is 1 .

Let us suppose that at least one firefighter is presented at some turn $k \leq$ $h(T)$; the tree still has at least one unburnt vertex. During the $(k-1)$ first turns, the instance is updated into $\left(B^{k}(T), r_{B^{k}},\left(f_{i+k}\right)\right)$ with one firefighter presented during the first turn and the root has at least one child. Proposition 1 ensures that it is equivalent to the original instance.

If the root has only one child $a=b$ at line 5 and algorithm 1 selects $a$ and saves all unburnt vertices inducing a competitive ratio of 1 . 


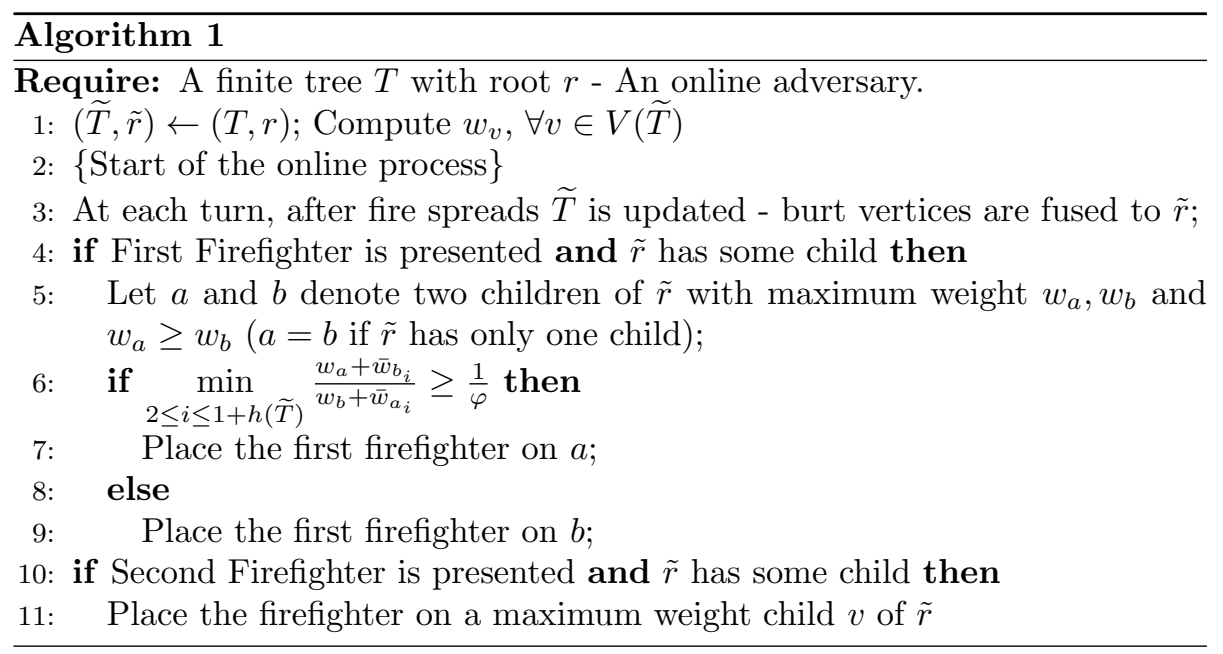

Else, $a \neq b$ with $w_{a} \geq w_{b}$ (line 5). Suppose first that the adversary presents a single firefighter during the whole process, then algorithm 1 places him on $a$ or $b$ while $B o b$ places him on $a$, saving $w_{a}$. If $w_{a} \geq \varphi w_{b}$, then we have:

$$
\forall i, 2 \leq i \leq 1+h(T), \frac{w_{a}+\bar{w}_{b_{i}}}{w_{b}+\bar{w}_{a_{i}}} \geq \frac{w_{a}}{w_{b}+w_{a}} \geq \frac{\varphi w_{b}}{w_{b}+\varphi w_{b}}=\frac{1}{\varphi}
$$

Therefore, (see line 6) the unique firefighter is placed on $a$ by the algorithm, guaranteeing a competitive ratio of 1 . Else we have $w_{b}>\frac{1}{\varphi} w_{a}$ and even placing the firefighter on $b$ guarantees the ratio $\frac{1}{\varphi}$.

Suppose now that the adversary presents two firefighters. We consider two cases.

Case (i): If algorithm 1 places the first firefighter on $a$ in line 7 and if the adversary presents the second firefighter at turn $i$, then the algorithm will save $w_{a}+\bar{w}_{x_{i}}$ for some $x \in T_{1} \backslash\{a\}$ such that $\bar{w}_{x_{i}}=\max _{u \in T_{1} \backslash\{a\}} \bar{w}_{u_{i}}$. For the same instance $B o b$ will save $w_{v}+\bar{w}_{y_{i}}$ for some $v \in\{a, b\}$ and $y \in T_{1} \backslash\{v\}$. If both solutions are not of the same value (the optimal one is strictly better), then necessarily $v=b$ and $y=a$. In this case the criterion of line 6 ensures that the related competitive ratio is $\frac{1}{\varphi}$.

Case (ii): Suppose now algorithm 1 places the first firefighter on $b$ in line 9, and say the adversary presents the second firefighter at turn $j$. Line 5 ensures that:

$$
\exists i, 2 \leq i \leq 1+h(T), \frac{w_{a}+\bar{w}_{b_{i}}}{w_{b}+\bar{w}_{a_{i}}}<\frac{1}{\varphi}
$$

It implies in particular that $w_{a}<\varphi w_{b}$ since in the opposite case eq. (3) would hold. In case (ii), algorithm 1 saves $w_{b}+\bar{w}_{x_{j}}$ with $x \in T_{1} \backslash\{b\}$ such that $\bar{w}_{x_{j}}=\max _{u \in T_{1} \backslash\{b\}} \bar{w}_{u_{j}}$. Meanwhile, Bob selects $v \in\{a, b\}$ and, if it exists, $y_{j}$ for some $y \in T_{1} \backslash\{v\}$, for a total of $w_{v}+\bar{w}_{y_{j}}$ saved vertices. If $y \neq b$, then $\bar{w}_{y_{j}} \leq \bar{w}_{x_{j}}$, by definition of $x$, and thus: 


$$
\frac{w_{b}+\bar{w}_{x_{j}}}{w_{a}+\bar{w}_{y_{j}}} \geq \frac{w_{b}+\bar{w}_{x_{j}}}{w_{a}+\bar{w}_{x_{j}}} \geq \frac{w_{b}}{w_{a}}>\frac{1}{\varphi}
$$

Finally, if $y=b$, then $v=a$ and the competitive ratio to evaluate is $\frac{w_{b}+\bar{w}_{x_{j}}}{w_{a}+\bar{w}_{b_{j}}}$. We claim that the following holds:

$$
\frac{w_{a}+\bar{w}_{b_{i}}}{w_{b}+\bar{w}_{a_{i}}} \times \frac{w_{b}+\bar{w}_{x_{j}}}{w_{a}+\bar{w}_{b_{j}}} \geq \frac{1}{\varphi^{2}}
$$

If $i \geq j$ we have $\bar{w}_{a_{i}} \leq \bar{w}_{a_{j}}$ and since $a \neq b, \bar{w}_{a_{j}} \leq \bar{w}_{x_{j}}$. Hence: $\frac{w_{b}+\bar{w}_{x_{j}}}{w_{b}+\bar{w}_{a_{i}}} \geq \frac{w_{b}+\bar{w}_{x_{j}}}{w_{b}+\bar{w}_{a_{j}}} \geq 1$ and therefore:

$$
\frac{w_{a}+\bar{w}_{b_{i}}}{w_{b}+\bar{w}_{a_{i}}} \times \frac{w_{b}+\bar{w}_{x_{j}}}{w_{a}+\bar{w}_{b_{j}}} \geq \frac{w_{a}+\bar{w}_{b_{i}}}{w_{a}+\bar{w}_{b_{j}}} \geq \frac{w_{a}}{w_{a}+w_{b}} .
$$

If now $i<j$, we get: $\frac{w_{a}+\bar{w}_{b_{i}}}{w_{a}+\bar{w}_{b_{j}}} \geq 1$ and therefore:

$$
\frac{w_{a}+\bar{w}_{b_{i}}}{w_{b}+\bar{w}_{a_{i}}} \times \frac{w_{b}+\bar{w}_{x_{j}}}{w_{a}+\bar{w}_{b_{j}}} \geq \frac{w_{b}+\bar{w}_{x_{j}}}{w_{b}+\bar{w}_{a_{i}}} \geq \frac{w_{b}}{w_{a}+w_{b}} .
$$

In both cases, since $\frac{w_{a}}{w_{a}+w_{b}} \geq \frac{w_{b}}{w_{a}+w_{b}} \geq \frac{1}{1+\varphi}=\frac{1}{\varphi^{2}}$ we deduce eq. (6). Now, eqs. (4) and (6) imply that in case (ii), when $y=b$, we also have $\frac{w_{b}+\bar{w}_{x_{j}}}{w_{a}+\bar{w}_{b_{j}}} \geq \frac{1}{\varphi}$. Together with eq. (5), this concludes case (ii) and shows that algorithm 1 is $\frac{1}{\varphi}$-competitive.

Even though complexity analyses are not usually proposed for online algorithms, it is worth noting that line 6 only requires the weights of vertices in $V(T[a]) \cup V(T[b])$ and the maximum weight per level in $T[a]$ and $T[b]$ and consequently Algorithm 1 requires $O(|V(T[a])|+|V(T[b])|)$ to choose the position of the first firefighter and $O(|V(T)|)$ in all.

We conclude this section with a hardness result justifying that the greedy algorithm $G r$ is optimal while Algorithm 1 is optimal if at most two firefighters are available.

Proposition 2. For all $k \geq 2, \frac{1}{2} \leq \alpha_{I, k} \leq \frac{1}{\varphi}$, more precisely:

(i) $\alpha_{I}=\frac{1}{2}$, meaning that the greedy algorithm is optimal for FIREFIGHTER in finite trees;

(ii) $\alpha_{I, 2}=\frac{1}{\varphi}$ meaning that Algorithm 1 is optimal if at most two firefighters are available;

(iii) $\alpha_{I, 4}<\frac{1}{\varphi}$.

Proof. Theorem 1 shows that $\alpha_{I} \geq \frac{1}{2}$. Given integers $l, m, k$ such that $k \mid m-1$, we define the graph $W_{k, l, m}$ as shown in fig. 1 . We will assume that $m>k^{2}$.

(i) Let us consider an online algorithm for $W_{k, l, m}$. If $f_{1}=1$, the algorithm will protect either $x$ or $y$. If $x$ is selected and the firefighter sequence is 


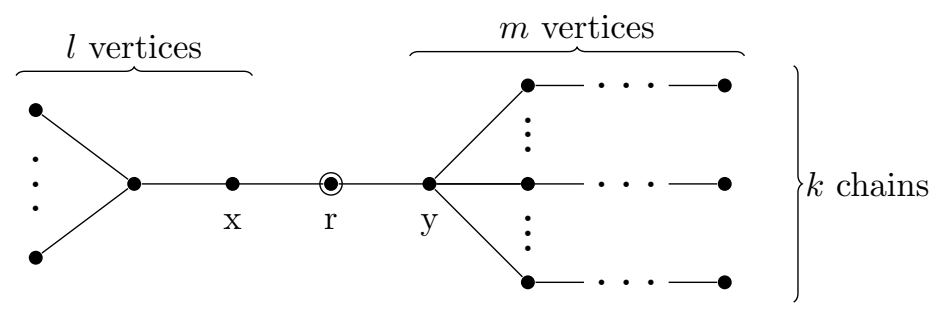

Figure 1: The fire on graph $W_{k, l, m}$ starts at vertex $r$. Whether the firefighter should protect $x$ or $y$ on the first turn depends on the firefighter sequence.

$(1,1,0,0, \ldots)$, our online algorithm protects the branch of $x$ and one of the $k$ chains, while the optimal offline algorithm protects $y$ and the star. Its performance is then $\frac{l+\frac{m-1}{k}}{l+m-1}$. If, however, $y$ is protected instead during the first turn and if the firefighter sequence is $(1,0,1,1, \ldots)$, the online algorithm protects the branch of $y$ and one vertex of the star whilst the optimal algorithm protects the branch of $x$ as well as the $k$ chains, minus for $\frac{k(k+1)}{2}$ vertices. If $l=m$, for large values of $m$, the online algorithm which protects $x$ is more performant and its competitive ratio tends to $\frac{1+\frac{1}{k}}{2}$. Considering, for instance, $W_{k, k^{3}, k^{3}}$, when $k \rightarrow+\infty$ shows that $\alpha \leq \frac{1}{2}$. Since the greedy algorithm $G r$ guarantees $\alpha_{I} \geq \frac{1}{2}$ we have $\alpha_{I}=\frac{1}{2}$.

(ii) Consider the sequence of graphs $W_{1, l,\lfloor\varphi l\rfloor}$. If the online algorithm protects $x$, the adversary selects the sequence $(1,0, \ldots)$, whereas if the online algorithm protects $y,(1,0,1,0, \ldots)$ is selected. In both cases, the performance tends to $\frac{1}{\varphi}$ when $l \rightarrow+\infty$.

(iii) If at most 4 firefighters are available, the graph $W_{4,901,1001}$ (fig. 1) gives an example where $\frac{1}{\varphi}$ cannot be reached. Ideed, if $f_{1}=1$ and the online algorithm protects $x$, then the adversary will select the sequence $(1,1,0, \ldots)$, as in the proof $(i)$, for a performance of $\frac{1151}{1901}$. If the online algorithm protects $y$, since firefighters are limited to 4 , the adversary will select $(1,0,1,1,1,0, \ldots)$, for a performance of $\frac{1002}{1645}$. This second choice is slightly better, but $\frac{1002}{1645}<\frac{1}{\varphi}$.

We have also proved that Theorem 2 holds if three firefighters are presented (i.e., $\alpha_{I, 3}=\frac{1}{\varphi}$ ). However, the proof involves a much more technical case-by-case analysis and will not be detailed here.

\section{Firefighting on Trees with Linear Growth}

In this section, we consider infinite trees. We say that a rooted tree $(T, r)$ has linear growth if the number of vertices per level increases linearly, i.e. $\left|T_{i}\right|=$ $\mathcal{O}(i)$.

Remark 1. The linear growth property of $T$ remains if we choose a different 
root $r^{\prime}$. Indeed, if $d$ is the distance between $r$ and $r^{\prime}$, the set of vertices at distance $i$ from $r^{\prime}$ is included in $\bigcup_{j=i-d}^{i+d} T_{j}$, the cardinal of which is a $\mathcal{O}(i)$.

Given two firefighter sequences $\left(f_{i}\right)$ and $\left(f_{i}^{\prime}\right)$, we say that $\left(f_{i}\right)$ is stronger than $\left(f_{i}^{\prime}\right)$ if for all $k, \sum_{i=1}^{k} f_{i} \geq \sum_{i=1}^{k} f_{i}^{\prime}$.

Lemma 2. If the fire can be contained in an instance $\left(G, r,\left(f_{i}^{\prime}\right)\right)$ and if $\left(f_{i}\right)$ is stronger than $\left(f_{i}^{\prime}\right)$, then the fire can also be contained in $\left(G, r,\left(f_{i}\right)\right)$.

Proof. Given a winning strategy in the instance $\left(G, r,\left(f_{i}^{\prime}\right)\right)$, if $\left(f_{i}\right)$ firefighters are available, we contain the fire by protecting the same vertices, eventually earlier than in the initial strategy.

Theorem 3. Let $\left(T, r,\left(f_{i}\right)\right)$ be an online instance of FraCtional Firefighter. If $T$ has linear growth and $\left(f_{i}\right)$ is stronger than some non-zero periodic sequence, then the fire can be contained.

The proof of theorem 3 will use the following lemma:

Lemma 3. For any $a>0, \lim _{n \rightarrow+\infty} \prod_{j=1}^{n} \frac{a j-1}{a j}=0$

Proof. We have $\ln \prod_{j=1}^{n} \frac{a j-1}{a j}=\sum_{j=1}^{n} \ln \left(1-\frac{1}{a j}\right)$ and $\sum_{j=1}^{n} \ln \left(1-\frac{1}{a j}\right) \sim-\sum_{j=1}^{n} \frac{1}{a j}$. So $\sum_{j=1}^{n} \ln \left(1-\frac{1}{a j}\right) \rightarrow-\infty$ and $\prod_{j=1}^{n} \frac{a j-1}{a j} \rightarrow 0$.

We can now prove theorem 3:

Proof. Since $T$ has linear growth, let $C$ be such that $\forall i,\left|T_{i}\right| \leq C i$. For all $n$, let $\left(\delta_{n \mid i}\right)$ denote the firefighter sequence where one firefighter is available every $n$ turns; i.e. $\delta_{i}^{n}$ is equal to 1 if $n \mid i$ and 0 otherwise. That the sequence $\left(f_{i}\right)$ is stronger than a non-zero periodic sequence means that $\left(f_{i}\right)$ is stronger than $\left(\delta_{n \mid i}\right)$, for all $n$ greater than some $m$. First we will give an offline strategy to contain the fire with one firefighter every $n$ turns. Then, we will show that online instances with $\left(f_{i}\right)$ stronger than a $\left(\delta_{n \mid i}\right)$ known to the player are winning. Finally, we will describe the winning strategy when such a $\left(\delta_{n \mid i}\right)$ is unknown.

Given an integer $n$, let us first consider the instance $\left(T, r,\left(\delta_{n \mid i}\right)\right)$. It follows from lemma 3 that there exists an integer $N$ such that $\prod_{j=1}^{N} \frac{C n j-1}{C n j}<\frac{1}{2 C n}$. Let $h(n)=2 n N$. A winning strategy is obtained by protecting at turn $n j$ the unprotected vertex of $T_{n j}$ with the highest number of descendants in level $h(n)$. Since $\left|T_{n j}\right| \leq C n j$, the remaining number of unprotected vertices in $T_{h(n)}$ is reduced by at least $\frac{1}{C n j}$ of its previous value. So the number of unprotected vertices of $T_{h(n)}$ remaining after $n N$ turns is less than $\left|T_{h(n)}\right| \prod_{i=1}^{N} \frac{C n j-1}{C n j} \leq$ $\frac{\left|T_{h(n)}\right|}{2 C n} \leq N$. Since $N$ firefighters remain to be placed between turns $N$ and $h(n)$, the strategy is winning in at most $h(n)$ turns.

If the player knows that $\left(f_{i}\right)$ is stronger than $\left(\delta_{n \mid i}\right)$, the above strategy can be adapted using lemma 2 .

In the general case, the player knows that $\left(f_{i}\right)$ is stronger than $\left(\delta_{n \mid i}\right)$ for some $n$, but he does not know which $n$. The strategy proceeds as follows: we 
initially play as though under the assumption that $\left(f_{i}\right)$ is stronger than $\left(\delta_{n_{0} \mid i}\right)$, with $n_{0}=100$. If the fire is not contained by turn $h\left(n_{0}\right)$, or later on by turn $h\left(n_{k}\right)$, we choose $n_{k+1}=h\left(n_{k}\right)\left(\left\lceil S_{h\left(n_{k}\right)}\right\rceil+1\right)$, where $S_{n}=\sum_{i=1}^{n} f_{i}$. We now assume that $\left(f_{i}\right)$ was stronger than $\left(\delta_{h\left(n_{k}\right) \mid i}\right)$. It follows that after cancelling the first $h\left(n_{k}\right)$ terms of $\left(f_{i}\right)$, the resulting sequence is stronger than $\left(\delta_{n_{k+1} \mid i}\right)$. So we can consider that the first $h\left(n_{k}\right)$ turns were wasted and follow the strategy for $n_{k+1}$ until turn $h\left(n_{k+1}\right)$. Eventually, this strategy will win when $n_{k}$ is large enough.

\section{Final remarks}

In this paper, we introduce the online version of (FraCtional) Firefighter and propose first results for both the finite and the infinite cases. To our knowledge, theorem 1 is the first non trivial competitive (but also approximation) analysis for Fractional Firefighter and a first question would be to investigate whether a better competitive ratio can be obtained for FRACTIONAL FirEFighter in finite trees. Even though the case of trees is already challenging despite allowing many simplifications, the main open question will be to study online (Fractional) Firefighter problem in other classes of finite graphs.

\section{References}

[1] Hartnell, B.: Firefighter! An application of domination. (1995) presented at the 10th Conference on Numerical Mathematics and Computing, University of Manitoba in Winnipeg, Canada.

[2] Finbow, S., MacGillivray, G.: The firefighter problem: a survey of results, directions and questions. Australasian Journal of Combinatorics 43(6) (2009) $57-77$

[3] Finbow, S., King, A., Macgillivray, G., Rizzi, R.: The firefighter problem for graphs of maximum degree three. Discrete Mathematics 307(16) (2007) 2094-2105

[4] Fomin, F.V., Heggernes, P., van Leeuwen, E.J.: The firefighter problem on graph classes. Theoretical Compututer Science 613(C) (February 2016) $38-50$

[5] Cai, L., Verbin, E., Yang, L.: Firefighting on trees: (1-1/e)-approximation, fixed parameter tractability and a subexponential algorithm. In: Algorithms and Computation, 19th International Symposium, ISAAC 2008, Gold Coast, Australia, December 15-17, 2008. Proceedings. (2008) 258269 
[6] Bazgan, C., Chopin, M., Ries, B.: The firefighter problem with more than one firefighter on trees. Discrete Applied Mathematics 161(7-8) (2013) 899-908

[7] Bazgan, C., Chopin, M., Cygan, M., Fellows, M.R., Fomin, F.V., van Leeuwen, E.J.: Parameterized complexity of firefighting. Journal of Computer and System Sciences 80(7) (2014) 1285-1297

[8] Hartnell, B., Li, Q.: Firefighting on trees: How bad is the greedy algorithm? Congressus Numerantium (2000) 187-192

[9] Adjiashvili, D., Baggio, A., Zenklusen, R.: Firefighting on trees beyond integrality gaps. In: Proceedings of the Twenty-Eighth Annual ACM-SIAM Symposium on Discrete Algorithms, SODA 2017, Barcelona, Spain, Hotel Porta Fira, January 16-19. (2017) 2364-2383

[10] Anshelevich, E., Chakrabarty, D., Hate, A., Swamy, C.: Approximability of the firefighter problem - computing cuts over time. Algorithmica 62(1-2) (2012) 520-536

[11] Hartke, S.G.: Attempting to narrow the integrality gap for the firefighter problem on trees. In: Discrete Methods in Epidemiology. (2004) 225-232

[12] MacGillivray, G., Wang, P.: On the firefighter problem. Journal of Combinatorial Mathematics and Combinatorial Computing 47 (2003) 83-96

[13] Fogarty, P.: Catching the Fire on Grids, PhD thesis. University of Vermont (2003)

[14] Ausiello, G., Becchetti, L.: On-line algorithms. In Paschos, V.T., ed.: Paradigms of Combinatorial Optimization: Problems and New Approaches, Vol. 2. ISTE - WILEY, London - Hoboken (2010) 473-509

[15] Chalermsook, P., Vaz, D.: New integrality gap results for the firefighters problem on trees. In Jansen, K., Mastrolilli, M., eds.: Approximation and Online Algorithms, Cham, Springer International Publishing (2017) 65-77 